\title{
Crown ether containing polyelectrolyte multilayer membranes for lithium recovery
}

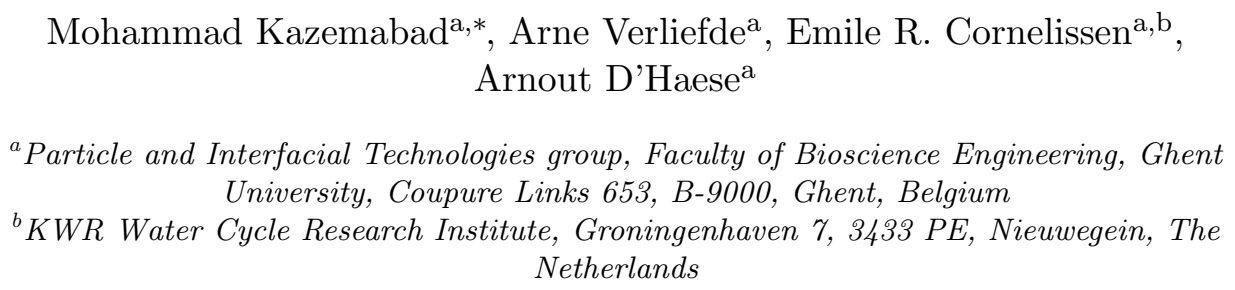

\section{Abstract}

Achieving solute selectivity has always been a goal of membrane development studies. The continuing growth of global consumption of scarce metals by different industries has put a strain on traditional sources of these species. Achieving cation selectivity in membranes, especially among monovalent cations, is a major step in introducing alternative sources for scarce metals such as lithium.

Polyelectrolyte multilayer membranes (PEMMs) are a novel class of membranes, offering great potentials in monovalent/bivalent ion selectivity. On the other hand, crown ethers are a well-studied family of macrocyclic ligands capable of forming stable complexes with cations. In the current study, for the first time, we report on a PEMM nanofiltration membrane with crown ether moieties embedded in its structure for the goal of achieving monovalent salt selectivity. The crown ether 15 -crown-5 was successfully incorporated in the polycation polyethylenimine (PEI), which was then used as the polycation in PEMM formation through layer by layer deposition. Both the synthesized polymer and the polyelectrolyte multilayer (PEM) were characterized and the performance of the resulting membrane

\footnotetext{
${ }^{*}$ Corresponding author 1

Email addresses: mohammad.kazemabad@ugent.be (Mohammad Kazemabad ), Arne.verliefde@ugent.be (Arne Verliefde), Emile.Cornelissen@kwrwater.nl (Emile R. Cornelissen), arnout.dhaese@ugent.be (Arnout D'Haese)
} 

was studied. It was determined that crown ether containing polymer forms more stable complexes with lithium than potassium. This was explained

29 by the limitation put on 2:1 potassium-crownether complexes by steric hin30 drance from polymer chain. The manufactured membranes showed Li/K 31 selectivity for a period of around 90 minutes, after which the crown ethers became saturated and selectivity was lost. The modified membranes became

33 non selective after this point, but possessed high salt rejection potential.

34 Keywords: polyelectrolyte multilayer membranes, layer by layer 


\section{Introduction}

37 Application of lithium in Li-ion batteries and the continuous shift from fossil-based energies towards electrical energy, has put a high pressure on conventional lithium resources. Based on the conservative estimations, current lithium reserves are projected to run out by 2080 [1]. Alternative 41 lithium sources such as sea water, industrial and mining waste waters, RO brines and spent lithium batteries can significantly lower the toll on the current reserves[2]. For example, assuming a recovery efficiency of $80 \%$, 44 more than $60 \%$ of the worldwide lithium consumption can be recovered 45 from RO desalination brine[3]. Lately, tremendous amount of effort has

been directed at the search for novel lithium sources and recovery methods by both academia and industry. Diallo and co-workers [3] have reviewed prospects of critical metal recoveries from seawater. Meshram et al. [4] have investigated traditional and novel methods of lithium recovery from primary and secondary sources. Although recovery from alternate sources is still very limited in an industrial scale, a number of small scale plants aimed at lithium harvesting from the seawater exist. For example the Institute of ocean energy at Saga University in Japan prepared a lab scale set-up for lithium extraction from seawater based on $\mathrm{MnO}_{2}$ adsorbers [4, 5]. In another attempt, Korea institute of geoscience and mineral resources (KIGAM) partnered with steel making industry POSCO in 2010 to launch a seawater lithium harvesting plant with the aim of reaching 30 tonnes of production over a course of four years. [6]

The greatest obstacles of lithium harvesting from alternative sources are the low concentration of lithium, and the presence of other alkali metals. Therefore development of a separation process capable of differentiat- 
ing among different monovalent cations is of great importance. Absorbents

of ion sieve type (such as mangenese oxide $\mathrm{H}_{1.6} \mathrm{Mn}_{1.6} \mathrm{O}_{4}$ and spinel-type $\lambda-\mathrm{MnO}_{2}$ ) are one of the most promising solutions to the selectivity obstacle, with the later capable of concentrating lithium up to 400 times from a brine with high concentrations of sodium[4, 7]. However the purity of the extracted lithium can be still low $(\approx 33 \%)$ and sodium contamination can still be problematic. Crownether based adsorbents are another class of cation selective adsorbents studied.

Layer by layer (LBL) deposition of polyelectrolytes is a membrane fabrication method which results in formation of ultra-thin poly electrolyte layers as active layer. Compared to other thin film deposition methods, LBL assembly is extremely cheap, simple and versatile[8] and provides the possibility of controlling the film properties while offering excellent flexibility and fine tuning [9]. Therefore polyelectrolyte multilayer membranes (PEMMs) have enjoyed considerable interest in recent years. An overview of the LBL literature and the PEMMs can be found in reviews $[10,11,12]$. Since the main mechanism of ion rejection in PEMMs is based on charge rather than size, LBL assembeled membranes are known to reject divalent ions better than the monovalents [10], however monovalent selectivity remains challenging.

Crownethers are a family of macrocyclic ligands well known for their ability to form stable complexes with cations. Agreement between the crown ether cavity size and the cation size is the main factor affecting the stability of the cation-ligand complex formed. This was originally proposed by Pedersen [13] and later confirmed by X-ray crystallography [14]. However, it only provides a rule of the thumb, leaving out several other factors that can influence and even reverse the order of cation preference[15]. 
The mechanism of the complex formation in crownethers involves a complete or partial shedding of the solvation shell of the dissolved ions and subsequent solvation of the formed complex. Therefore the nature of the medium in which complexation is taking place plays a vital role in the thermodynamics of the reaction. It should be mentioned that in our case, the complexation is taking place inside the membrane pores, where an accurate definition of the medium is debated. It is known that physical properties of confined water in nanopores and especially its dielectric constant is different from that of the bulk water $[16,17]$. Moreover, hydration shells of the cations are prone to rearrangement or even breakage inside the nanopores [18]. It can thus be expected that crownethers confined in nanopores can act different than when they are free in the bulk.

Selectivity of the crownethers is also affected by the ring flexibility[15, 19]. Modifying the ring rigidity is regularly used to increase selectivity[19, 20]. The nature of the chemical groups in immediate vicinity to the ether groups also have a profound effect on the strength of the interaction. The electron density of the adjacent oxygen atoms and thus the binding capacity of the ligand are affected by attached groups. For example, it is shown that $\mathrm{K} / \mathrm{Na}$ selectivity can be reversed in crownethers with electron releasing or electron attracting substituents[15]. It is also shown that extremely stable complexes can be formed when there are anionic groups attached to the crownether[21]. Therefore, the cation-crownether complexes in the PEMMs, where anionic groups from the polyanion chain are present, are expected to be stronger. It's been reported that the response of different cations to the presence of the anionic groups is unpredictable and generalization is not possible [15].

Based on the relative size of the cation and the cavity, the formed com- 
plex can be of different stoichiometries. The 2:1 sandwich chemistry, in which the cation is present on top of one crownether and is sandwiched from the other side by another, provides the complex formation possibility for larger cations $[22,15]$. For example it is shown that in solid state, the potassium complex in benzo-15-crown-5 (the precursor used in this work) is of the sandwich type[23]. In some cases existence of such higher order chemistries compromises the selectivity potential of crownethers. Formation of this type of complexes can be limited by adding bulky subunits to the ether ring, blocking the formation of sandwich complexes by steric hindrance $[19,24,25,26]$. In a similar manner, polymerization of crownethers can affect their potential for forming higher order complexes. It is known that the order of cation preference changes in poly-crownethers with respect to their monomers. For example, Kimura et al. found that polymers of 18-crown-6 and 15-crown-5 have higher binding constants for cations that tend to form 2:1 complexes than their respective monomers, whereas binding constants of the smaller cations which form 1:1 complex remain more or less unchanged [27]. We expect that incorporation of the crownethers in highly branched PEI polymer in this work, introduces high steric hindrances and seriously limits the 2:1 complexing ability.

Considering all the above mentioned factors, it is inherent that the order of cation preference in the polymer synthesized in this project can't be predicted from its precursor crownethers. To the best knowledge of the writers, this is the first time this modification route is being taken for PEI as a polymer for membrane fabrication and thus no prior data is available on the complex strength of different cations and the crownether.

Crownethers have been used in several studies on membranes. However, in most cases, crownethers serve as the carrier in carrier mediated 
transports. To the best knowledge of the writers, this is the first report on usage of crownethers in a pressure driven nanofiltration membrane. Liquid membranes (LMs), a family of membranes where a liquid organic phase separates two aquatic phases, build up a substantial part of the literature on crownethers $[28,29,30,31]$. The most important problems of this class of membranes are leaching of the organic solvent or carrier and contamination of the aquatic phases due to the limited solubilities [32, 33, 34]. As a close relative to LMs, plasticized polymeric membranes reportedly house semi-liquid pockets in between their crystalline parts [35]. Crownethers have been used as fixed carriers in plasticized cellulose triacetate (CTA) for gold, silver, copper [34] and cesium [36, 37] ion transport.

Crown ethers have also been regularly used in ion exchange resins and membranes. The group of Bartsch developed several polycrownethers with acidic side arms to be used as ion exchange resins to achieve alkali metal [38, 39] and lead (II) [40] selectivity. Favre-Reguillon et al. [41] immobilized a number of crown ethers on polyurethans and studied the effect of polymer chain rigidity on the complexation. More recently, Tas and co-workers incorporated 18-crown-6 in Poly arylene ether ketone (PAEK) backbone and blended it with sulfonated poly ether ether ketone (SPEEK) to prepare monovalent cation selective membranes which they tested under diffusion dialysis [42].

Crownethers can be added to the membrane by either blending or covalent bonding to the matrix. Blending crownethers with the polymer solution during phase inversion or interfacial crystallization is one of the most common methods of embedding crown ethers in membrane structure. In the resulting membranes, crown ethers are trapped in the polymer matrix and therefore more immune to leaching [43]. Major disadvantages of this method 
are the promotion of structural defects and the possibility of demixing and uneven distribution [35]. Dulyea and co-workers prepared phase inverted membranes by blending a crownether containing polymer with an otherwise impermeable polymer. The resulted membranes showed a Na/K selectivity of 1.8:1. However, the membrane structure was disrupted at high crownether concentrations and defects in membrane structure even at low concentrations were reported [33]. Therefore, embedding of crownethers through covalent bonds in polymers is believed to yield more stable membranes. An overview of different methods to incorporate crownethers in polymers is given by Alexandratos and Stine [43].

Careful consideration must be given in selection of the crown ether moiety. Due to the close resemblance of the ionic radii and chemical properties of alkali metals, most of the crown ethers form complexes (with varying degrees of stability) with multiple ions. An optimal performance of the membrane can be achieved by selecting a crown ether which has a fundamentally different reaction to the desired target ion. In our case, this translates in forming complexes with all alkali metals except lithium. The complex formation constants from table 1 confirm such a property for 15-crown-5. Lithium15-crown-5 complexes are unstable in water [44], whereas potassium and sodium are capable of forming stable complexes [45, 46, 47, 48].

\subsection{Goal of the study}

PEMMs possess a natural monovalent/bivalent selectivity which makes them suitable for development of selective membranes. Monovalent selectivity can be introduced to these membranes by inclusion of crown ethers. Here, we report the covalent attachment of the crown ethers as pendant groups to the poly cation chain and subsequent LBL deposition of the modified 
Table 1: Thermodynamic data of complexes of cations with 15-crown-5 and benzo-15crown-5.

\begin{tabular}{c|c|c|c|c|c} 
Crownether & Cation & $\log \mathrm{K}_{\mathrm{a}}$ & Medium & Method & reference \\
\hline \multirow{4}{*}{ 15-crown-5 } & $\mathrm{Li}$ & $\approx 0$ & $\mathrm{H}_{2} \mathrm{O}$ & NMR & {$[44]$} \\
& $\mathrm{Na}$ & 0.44 & $\mathrm{H}_{2} \mathrm{O}$ & NMR & {$[47]$} \\
& $\mathrm{Na}$ & 0.70 & $\mathrm{H}_{2} \mathrm{O}$ & Calorimetry & {$[45]$} \\
& $\mathrm{K}$ & 0.74 & $\mathrm{H}_{2} \mathrm{O}$ & Calorimetry & {$[45]$} \\
\hline \multirow{3}{*}{ benzo-15-crown-5 } & $\mathrm{Na}$ & 0.40 & $\mathrm{H}_{2} \mathrm{O}$ & Electrophoresis & {$[48]$} \\
& $\mathrm{K}$ & 0.38 & $\mathrm{H}_{2} \mathrm{O}$ & Electrophoresis & {$[48]$} \\
& $\mathrm{K}$ & 0.38 & $\mathrm{H}_{2} \mathrm{O}$ & Calorimetry & {$[46]$} \\
\hline
\end{tabular}

polycation and polyanion to form a lithium selective nanofiltration membrane. It was crucial to investigate membrane stability at high salinities since PEMMs are known to be susceptible to high salinity and proposed alternative lithium sources such as RO brines are highly concentrated.

One of the important differences of the membrane prepared in this study and the majority of the crown ether containing membranes such as LMs is the nature of the matrix. The organic liquid phase of LMs are virtually impermeable to water and ions and crown ethers offer the only ion permeation pathway. Whereas normal PEMMs are permeable to water and ionic species. In our case, ion exchange sites intrinsic to the polyelectrolytes are much more abundant than crownethers. Therefore "ion hopping" can be expected to take place through these sites. Incorporation of the crownethers in the PEMM structure introduces a number of complexation sites in the membrane. Strong complexes formed in these sites can act as an Ion trap, hindering the transport of the cation across the membrane. The differences 
in the complex strength between different cations is reflected in the selectivity. Based on the complexation strength, trapped ions need to overcome a certain energy barrier to move past the membrane. Therefore we expect the membrane to hinder the transport of complexing cations. It can thus be expected that the membrane will reject the complex forming ions more, and cations with the less stable complexes will be more abundant in the permeate. Based on the thermodynamic data (Table 1), we expect a reduction in the transport rate of all alkali metals in the crown ether containing membranes. However, this reduction will be more severe in the case of sodium and potassium in comparison to lithium, resulting in a lithium rich permeate.

\section{Materials and methods}

\subsection{Synthesis and characterization of crownether incorporated polycation}

\subsubsection{4'formylbenzo 15 crown-5}

4'Formylbenzo 15-crown-5 was synthesized by a ring closure reaction between a linear ether and 3,4-dihydroxybenzaldehyde based on the method of Ungaro et al.[49] with slight modifications. A detailed explanation of the synthesis procedure can be found in the supporting information. In total, 3.9 grams of crownether crystals were purified, corresponding to a yield of around $20 \%$. The low yield of the procedure is attributed to two factors: undesired reactions and loss of product during purification steps. Of these, the former is responsible for almost half of the loss. Possible side reactions include monosubstitued reaction of the hydroxyl groups of dihydroxybenzaldehyde resulting in an open ring and the association of the benzaldehyde to two different ether chains. 


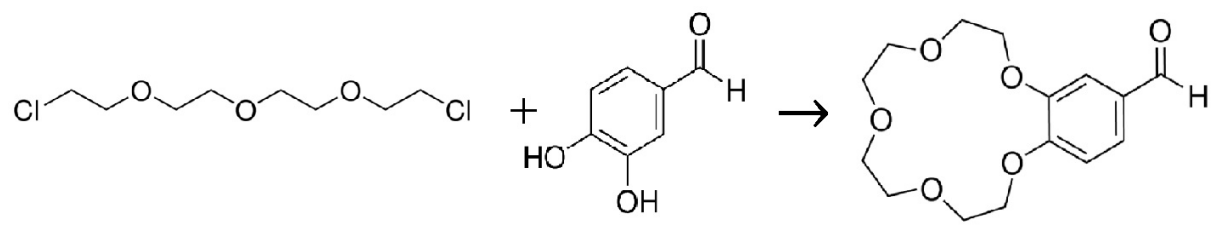

Figure 1: synthesis route for 4'formylbenzo-15crown5.

The nature and purity of the resulting crystal was studied by NMR spectra. HNMR was performed at $400 \mathrm{MHz}$ with a Bruker Avance III Nanobay $400 \mathrm{MHz}$ spectrometer. Tetramethylsilane (TMS) was used as reference compound. It was determined that the final crystals had a purity of about 95\%. The main remaining impurity was unreacted linear ether which was not removed during the purification, with HNMR peaks between 3.6 and 3.8 ppm.

\subsubsection{Incorporation of crown ether in PEI}

The synthesized formylbenzo 15 crown- 5 was incorporated in the Polyethylenimine (PEI) structure by reductive amination. The protocol was adopted from the work of Kasprzak et al. who added chemically similar p-formylbenzoic acid to PEI [50]. The PEI solution (50 wt\% in water, highly branched, $\mathrm{M}_{\mathrm{w}}$ $750 \mathrm{kDa}, \mathrm{M}_{\mathrm{n}} 60 \mathrm{kDa}$, Sigma-Aldrich) was first dried using a rotavapor under vacuum. $50 \mathrm{ml}$ of methanol was then charged with $2.5 \mathrm{~g}$ of dry PEI, $15 \mathrm{mg}(0.25 \mathrm{mmol})$ of acetic acid and $375 \mathrm{mg}(1.25 \mathrm{mmol})$ of synthesized 4'-formylbenzo 15crown-5. The mixture was refluxed for one hour and then left to stir at room temperature for 24 hours. $190 \mathrm{mg}(5.0 \mathrm{mmol})$ of $\mathrm{NaBH}_{4}$ was then added to the mixture as the reducing agent and stirring continued for another 4 hours at room temperature. 


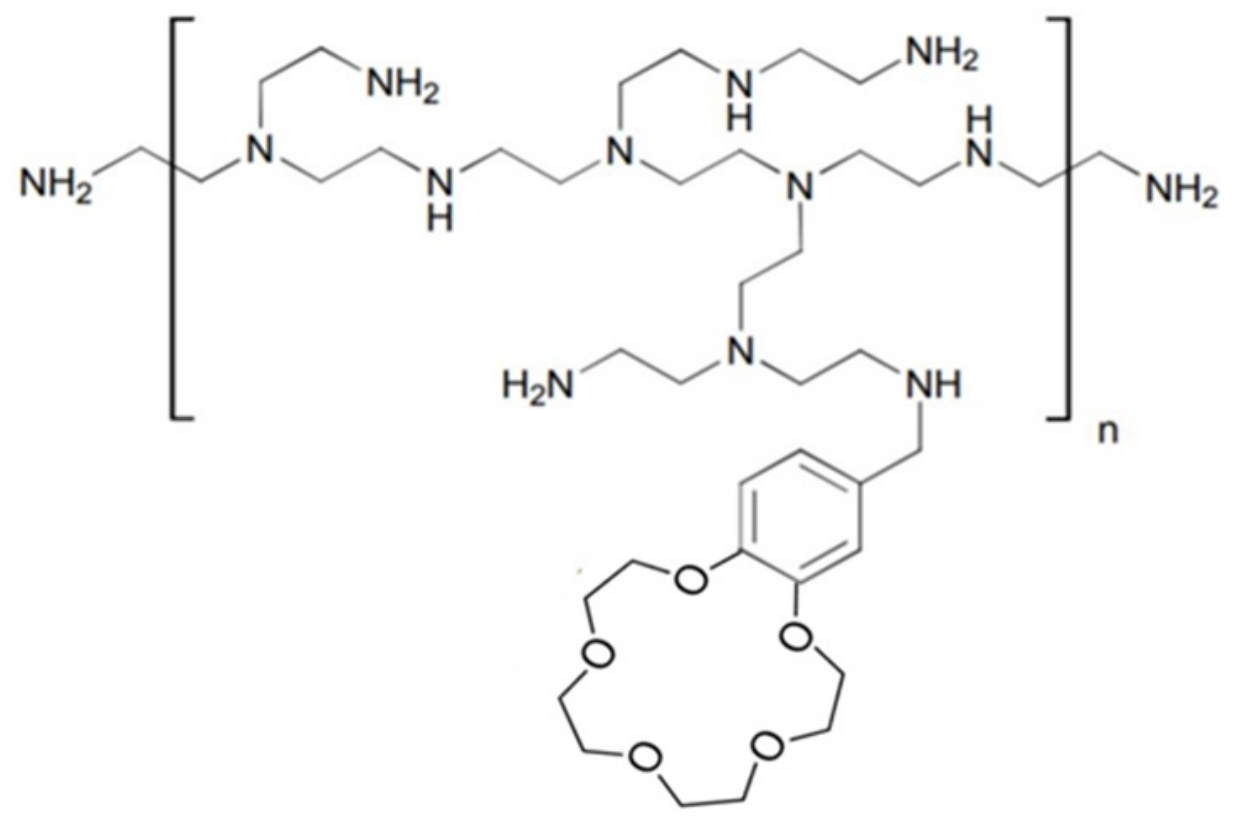

Figure 2: crownether incorporated polyetherimine.

\subsubsection{Polymer purification and characterization}

To remove the unbound crownethers and any other byproducts from modified PEI, the solution was extracted three times with ethyl acetate, leaving PEI in the aqueous phase. The resulting solution was then dialysed using DI water for two days. A Spectra/Por ${ }^{\circledR}$ regenerated cellulose dialysis membrane kit with MWCO of $12-14 \mathrm{kDa}$ from Spectrum labs (US) was used for this procedure. HNMR spectra (Fig. 3) validated the presence of crown ethers in the polymer structure. Peak were assigned to hydrogens with the help of ACDLabs's 1D NMR Processor software (ACD labs, Canada) (refer to Table.2). From these data, it was determined that crownether has been added in a ratio of one molecule for every 44 amine groups, corresponding to $0.53 \frac{\mathrm{mmol} \text { crownether }}{\mathrm{g} \text { of polymer }}$ (for a detailed discussion on calculation of this ratio 
refer to Appendix B in the supporting material). The stoichiometric ratios of reactants have a direct effect on the ratio of crown ether/PEI monomer in the final product, which in turn affects the performance of the synthesized membranes. However, only two recipes were tested and it was decided that an optimization of the crown ether/monomer ratio is out of the scope of the current study.

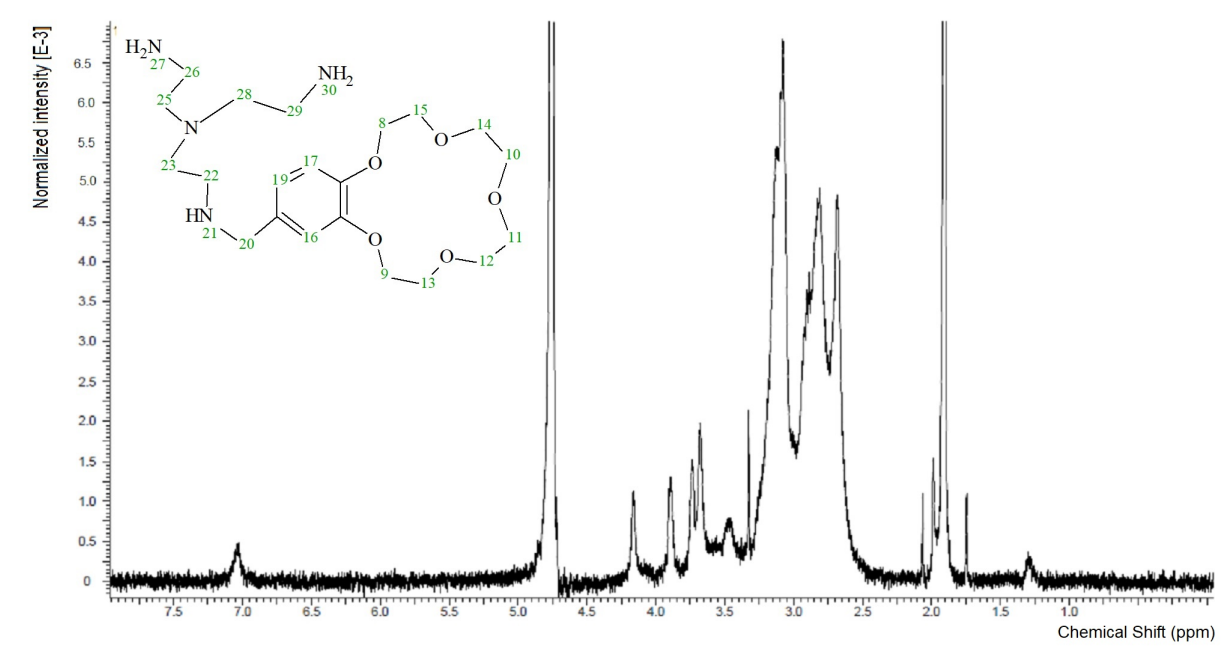

Figure 3: HNMR spectrum of crownether corporated polyetherimine in $\mathrm{D}_{2} \mathrm{O}$. please refer to 2 for list of assigned peaks. $\mathrm{X}$ axis represents chemical shift (ppm) and $\mathrm{Y}$ axis represents normalized intensity $\left(\times 10^{-3}\right)$

\subsection{Validation of PEM formation and evaluation of layer thickness via} $Q C M-D$

Highly branched polyethylenimine (PEI) was used as the polycation on this study. Poly styrenesulfonate (PSS), arguably the most common polyanion, was chosen to be paired with PEI. To validate the formation of the PEM, quartz crystal microbalance with dissipation (QCM-D) was used. The QCMD technique can determine the mass and physical properties of the film de- 
Table 2: Assigned peaks to the NMR spectrum

\begin{tabular}{c|c} 
Atom number & H shift $(\mathbf{p p m})$ \\
\hline $26,29,27,30$ & 1.905 \\
$23,25,28$ & 2.684 \\
22 & 2.807 \\
$12,14,10,11$ & 2.897 \\
$20,13,15$ & 3.079 \\
21 & 3.327 \\
8 & 3.679 \\
9 & 3.733 \\
19 & 7.027 \\
17,16 & 7.055
\end{tabular}

posited on the surface of the sensor by measuring the change of resonating frequency of a quartz crystal resonator. Measurements were performed on a Q-Sense E4 from Biolin scientific (Sweden), using silica coated quartz crystals with a fundamental frequency of $5 \mathrm{Mhz}$ (Biolin scientific-Sweden). A Rheodyne MX seriesII ${ }^{\mathrm{TM}}$ actuated valve from Idex health and science (US) was used as liquid handler for switching between different streams at the designated times.

The procedure of $\mathrm{PE}$ coating on the sensors was in close resemblance to the dip coating procedure used (refer to section 2.4). PSS $\left(\mathrm{M}_{\mathrm{w}} 70 \mathrm{kDa}\right.$, Sigma-Aldrich) was used as polyanion. A $0.4 \mathrm{wt} \% \mathrm{PE}$ solution in $0.5 \mathrm{M} \mathrm{NaCl}$ was pumped at $0.1 \mathrm{ml} / \mathrm{min}$ for $15 \mathrm{~min}$, followed by the rinsing steps at $0.5 \mathrm{M}$ $\mathrm{NaCl}$ in DI water. The duration and flow rate of the rinsing step was also $15 \mathrm{~min}$ and $0.1 \mathrm{ml} / \mathrm{min}$ respectively. Raw data were processed with the 
help of Qsense-Dfind software provided by Biolin Scientific. A density of 1.2 $\mathrm{gr} / \mathrm{cm}^{3}$ was assumed for the polyelectrolyte layers in accordance with the literature [51]. Each set of measurements was performed on four identical sets of sensors and results were averaged.

\subsection{PEM stability}

To assess the stability of the resulting PEM at high salinity levels, a test with QCM was designed. Stability of four set of PEMMs (modified and unmodified polycation, PEMMs ending in positively or negatively charged layers) was studied. Silica coated quartz crystals were coated with either four layers (2 bilayers) or five layers (2.5bilayers) of PEs. This step is marked in the Fig. 6 in supporting materials as PE coating step. At the end of the coating step, the sensors were rinsed with Milli-Q water to obtain a baseline. This is necessary since a change in the salinity results in change of the multilayer swelling and water content. The water content of the membranes are reflected in the QCM results, rendering the stability validation by comparison of membrane thicknesses in saline solutions impossible. Comparing the signals at Milli-Q water eliminates this effect. The multilayer was then put in contact with a saline solution of $\mathrm{NaCl}$ with varying concentration, starting from $1 \mathrm{M}$ and raised stepwise in $0.25 \mathrm{M}$ steps until reaching $4.75 \mathrm{M}$. Higher concentrations were not pursued due to the risk of $\mathrm{NaCl}$ crystallization inside the instrument. Adequate time was given to the PE multilayer to reach a constant frequency $(\Delta f / t<0.1 \mathrm{~Hz} / \mathrm{min})$. After this the solution was changed to Milli-Q to quantify mass loss from the PEM. These steps are identifiable in Fig. 5 and Fig.6 in supporting material with their respective $\mathrm{NaCl}$ concentrations. 


\subsection{Membrane synthesis via LBL assembly}

Nanofiltration membrane coupons were prepared via dip coating on a Polyacrylonitrile (PAN) support layer. A PAN ultrafiltration membrane from Synder filtration (US) with a MWCO of $30 \mathrm{kDa}$ was hydrolyzed by immersion in a solution of $\mathrm{NaOH} 1.5 \mathrm{M}$ at $45^{\circ} \mathrm{C}$ for 1.5 hours. Membrane coupons (with both sides exposed) were then immersed in subsequent solutions of $0.4 \mathrm{wt} \% \mathrm{PE}$ in the presence of $0.5 \mathrm{M}$ of $\mathrm{NaCl}$ as supporting electrolyte for 15 minutes. Rinsing was also performed using a $0.5 \mathrm{M} \mathrm{NaCl}$ solution for 15 minute periods. A complete cycle of bilayer deposition consisted of dipping the membranes in solutions of polycation, rinsing, polyanion and rinsing again. This cycle was repeated three times to obtain 6 layers (three bilayers) of poly electrolytes. The pH of the PSS solution was not adjusted, whereas the $\mathrm{pH}$ of the PEI was always adjusted to 9 for both modified and unmodified cases. After coating, the coupons were stored in DI water for at least 48 hours until use.

\subsection{Filtration experiments}

Dead-end filtration experiments were carried out to characterize the manufactured membranes. For this purpose, a $300 \mathrm{ml}$ Sterlitec $^{\mathrm{TM}} \mathrm{HP} 4750$ stirred cell connected to a nitrogen pressurized vessel was used. The experiments typically lasted for at least six hours, however, at least one experiment of each data set was continued for up to 48 hours to verify the stability of the results. The permeate was sampled more frequently at the beginning until steady state was reached, after which sampling frequency was reduced. Transmembrane pressure was set at five bars. The filtration vessel was kept under constant stirring at room temperature for the duration of the experiment. 
Single salt experiments using $\mathrm{LiCl}, \mathrm{NaCl}$ and $\mathrm{KCl}$ were performed at a feed cation concentration of $200 \mathrm{ppm}$. Mixed salt experiments were designed to investigate the effect of cation competition. It is known that the order of cation preference of crownethers can be different in competitive mode than the single cation mode [38]. A solution of $\mathrm{LiCl}$ and $\mathrm{KCl}$ with a cation concentration of $100 \mathrm{ppm}$ each, was used as feed for mixed salt experiments. Since the membranes were prepared in presence of $\mathrm{NaCl}$, they always contained some $\mathrm{NaCl}$ prior to first use. This made an accurate mass balance impossible and it was decided to do not include $\mathrm{NaCl}$ in the mixed salt experiments. In between the experiments, membrane coupons needed to be treated to regenerate the crownether sites. In most of the studies on absorbents and ion exchange resins this is done by acid treatment $[42,52]$. However PEMMs are unstable at extreme pHs and it was decided to regenerate membranes by saturating them with $\mathrm{Na}$ ions. This was done by filtering a $\mathrm{NaCl} 200$ ppm solution (similar to single salt $\mathrm{NaCl}$ experiments). Membrane coupons which were being used for the first time are designated as 'virgin' whereas all non specified specimen are regenerated coupons.

\section{Results and discussion}

\subsection{PEM formation}

Formation of a stable polyelectrolyte multilayer was confirmed by QCMD experiments in the case of both modified and unmodified PEI. Furthermore reaching a constant frequency $(\Delta f / t<0.1 \mathrm{~Hz} / \mathrm{min})$ before 15 minute confirmed the suitability of the time span selected for adsorption and rinsing steps (refer to section 2.2). The evolution of layer thickness was linear in the case of PEMs from unmodified PEI. This is in agreement with the 
data reported in literature [51]. Such a linear growth regime points to a low degree of intermixing between PE layers [53].

The evolution of PEM thickness was more complex in the case of modified PEI/PSS pair (Fig. 5). Instead of an increase in thickness upon addition of a PSS layer, the PEM lost part of its thickness (for example notice lower thickness of the second PSS layer in comparison to the second PEI layer in Fig. 5). This can be explained by lower degree of swelling of the membranes with PSS capping layers. It should be noted that the QCM-D method can not differentiate between the poly electrolyte mass and the water content of the PEM. Fig. 4 shows frequency shift and dissipation evolution during a typical coating procedure of the modified PEI/PSS multilayer. The dissipation value is consistently lower after PSS rinsing steps than PEI rinsing steps. Lower dissipation point to more rigid films [54] which in case of PEMs are usually result of lower swelling and less looped polyelectrolytes. It can thus be concluded that after addition of PSS layers, polymer films loose their water content and become less thick and more rigid.

Multilayers formed from modified PEI were consistently thicker in comparison to their unmodified counterparts. One of the possible explanation of this increased thickness can be the differences in PEM structure such as presence of polymer chain loops and PEM water content and swelling. The ratio of deprotonated amines to all amines in the modified PEIs is most likely to be different from the unmodified counterpart. Another possible explanation comes from the steric hindrance caused by relatively spacious crown ether molecules. Some of the positively charged sites on PEI can become inaccessible to the polyanion by this steric hindrance. Crown ethers limit approaching of the sulfonate groups on PSS to the nearby protonated amines. This reduces the apparent charge density of the PEI chain and 

studies.

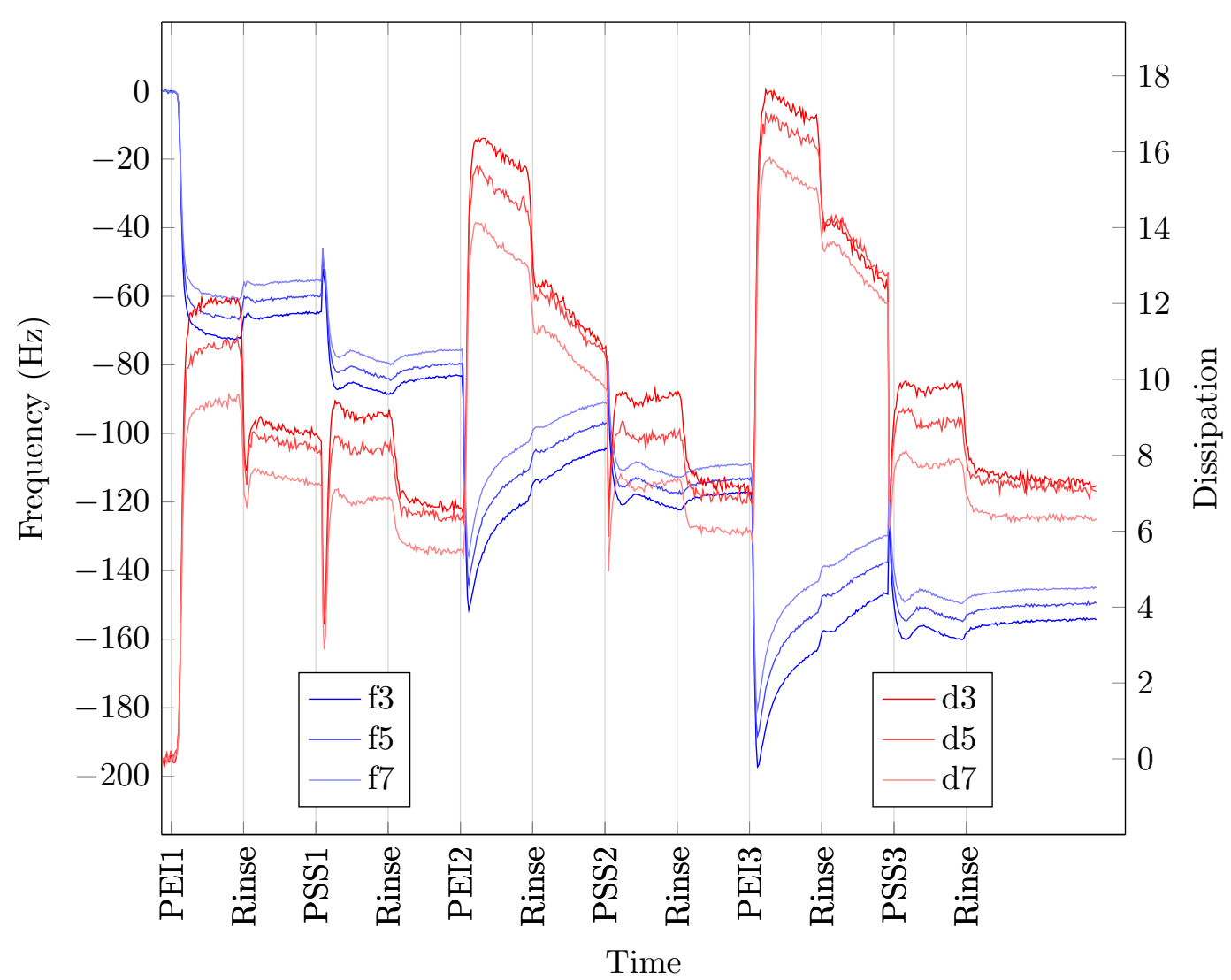

increases the amount of PEI needed to reach equilibrium and overcompensate. A definitive explanation of the nature of the increase in thickness can only come from a more thorough characterization of membranes in future

Figure 4: Frequency and dissipation vs. time of a typical modified PEI/PSS multilayer coating. For clarity only $3^{\text {rd }}, 5^{\text {th }}$ and $7^{\text {th }}$ overtones are shown. A similar plot for the unmodified PEI/PSS pair can be found in the supporting materials.

399

401 402

\subsection{PEM stability}

Stability of the PEM at high salinities was studied in four cases: PEMs prepared from modified and unmodified PEI and with positive or negative 


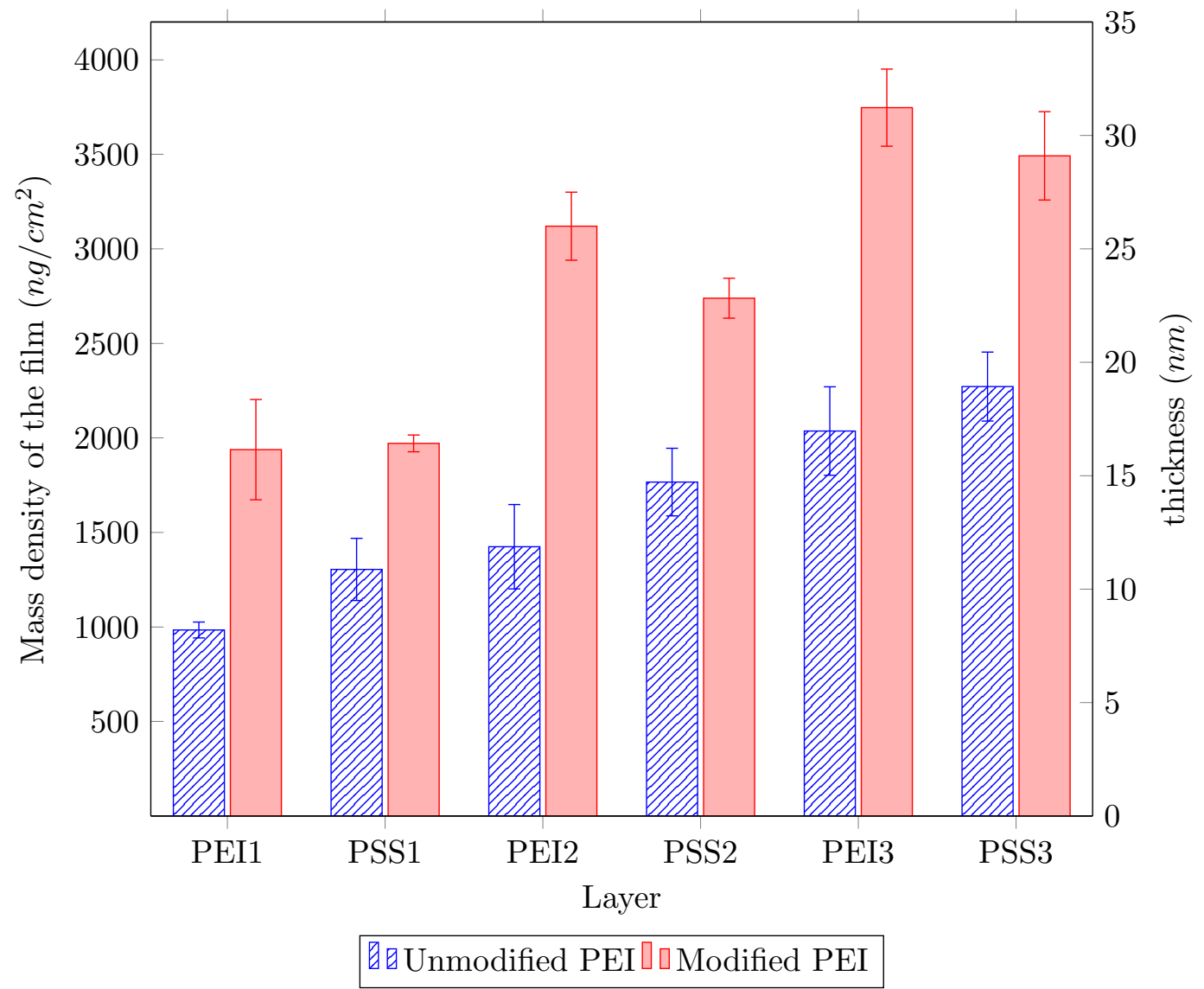

Figure 5: Evolution of thickness of layers as evaluated by QCM-D. Case of negative capping layer. The left axis represents the mass of the absorbed film per square $\mathrm{cm}$ of sensor. The right axis represents approximate thickness of the film based on the assumption of a density of $1.2 \mathrm{gr} / \mathrm{cm}^{2}$. Error bars represent standard error.

capping layer (final layer). These experiments were time consuming due to the long times needed to reach the constant signal, and the many steps involved (more than 72 hours for modified PEI/PSS PEMs). This rendered processing of the data with D-find software computationally intensive and not possible. As a result, change of the frequency ( $7^{\text {th }}$ overtone) was followed as a rough indicator of the adsorbed mass of the film. Changes in frequency 


$$
\Delta f / f_{0}=\frac{-\left(f-f_{0}\right)}{f_{0}} * 100 \%
$$

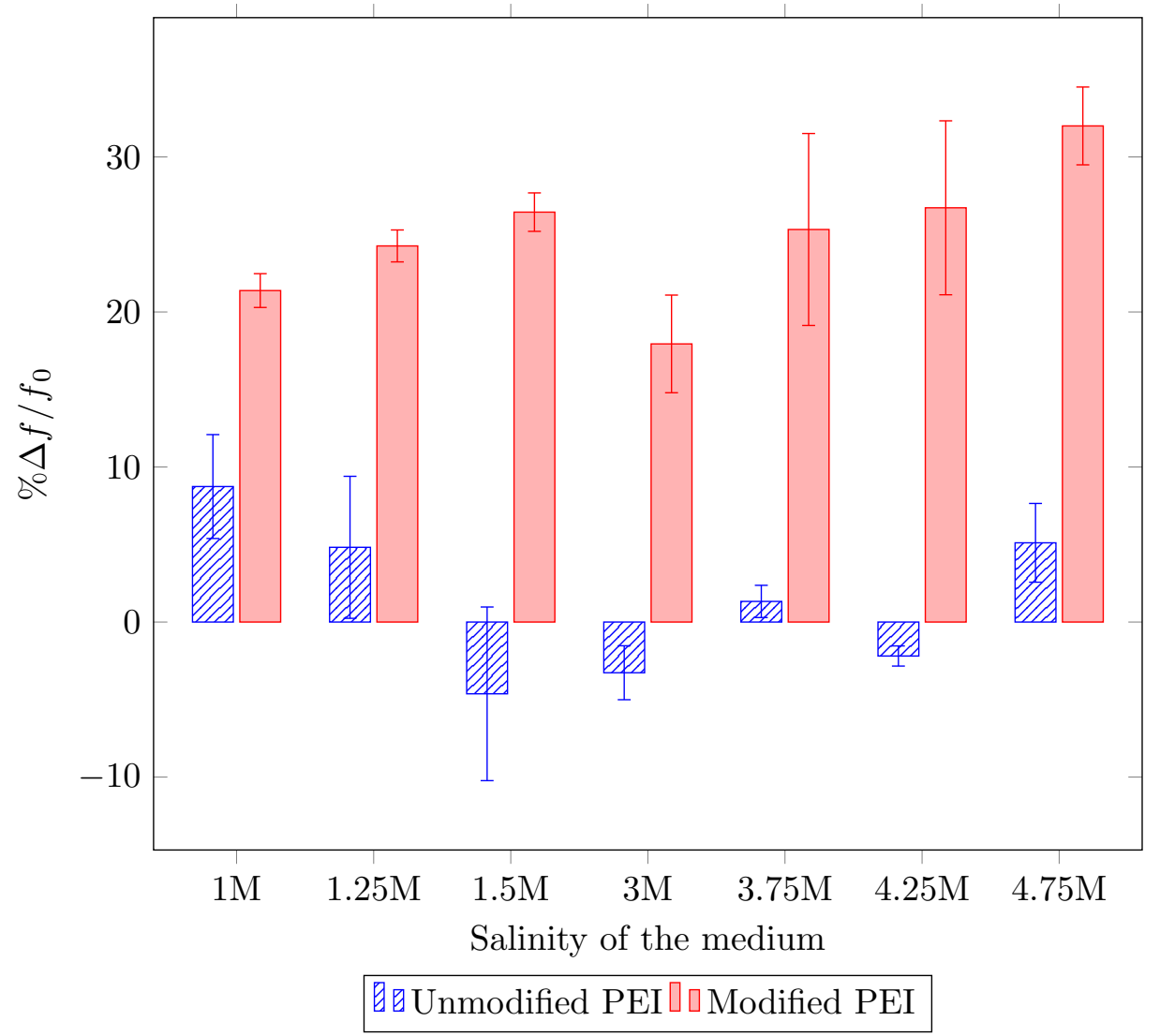

Figure 6: Percentage mass loss after contact with the saline solution versus salinity of the medium. Case of the negatively capped films (4 layers). Frequencies measured after reaching stable signals $(\Delta f / t<0.1 H z / \min )$. Error bars represent standard error from 4 samples. Some of the steps are not reported to enhance the clarity of the chart. phenomena: 


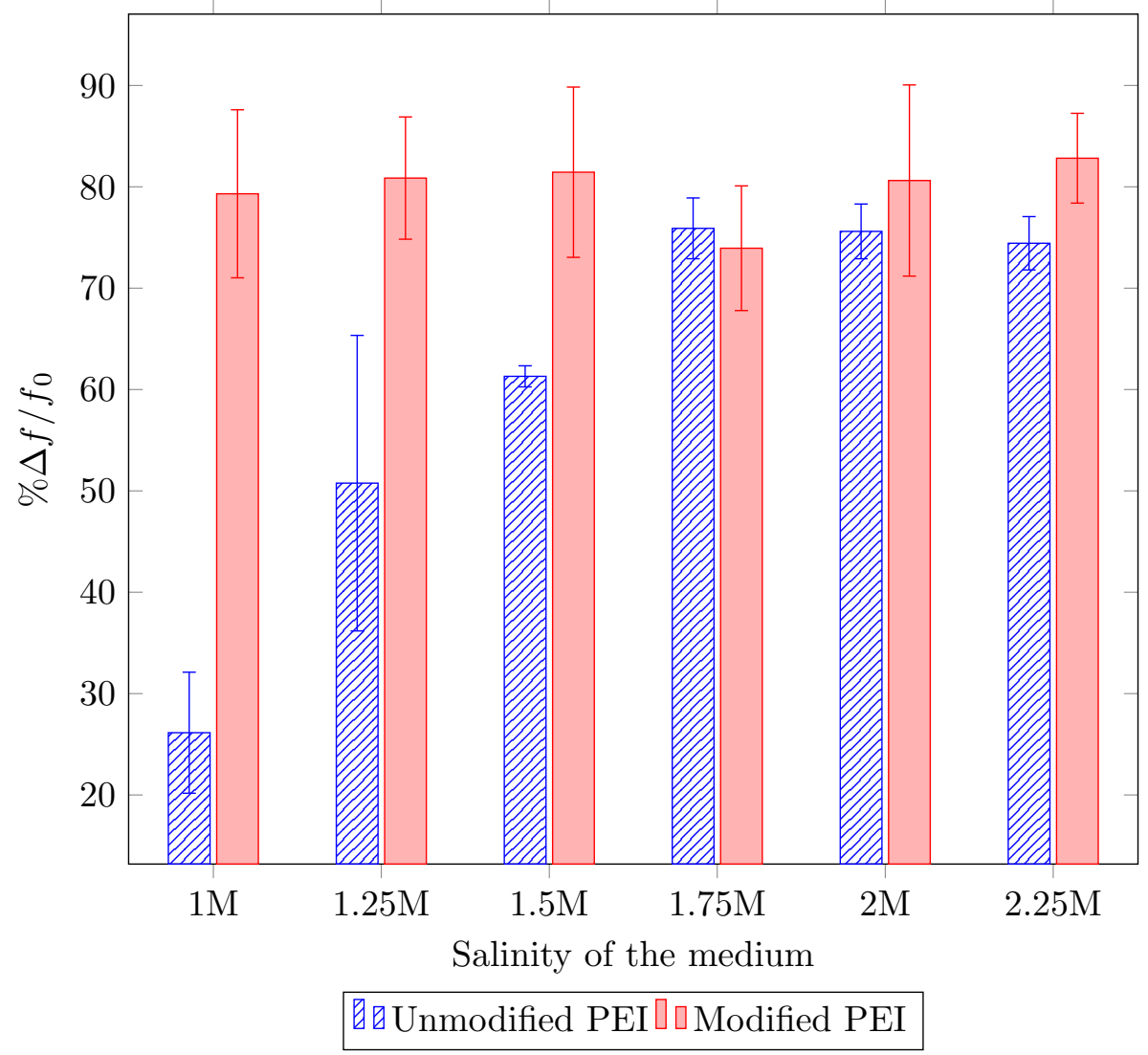

Figure 7: Percentage mass loss after contact with the saline solution versus salinity of the medium. Case of the positively capped films(5 layers). Frequencies measured after reaching stable signals $(\Delta f / t<0.1 \mathrm{~Hz} / \mathrm{min})$. Error bars represent standard error from 3 samples. Some of the steps are not reported to enhance the clarity of the chart.

I. Poly electrolyte detachment due to the instability,

II. Film water content due to the internal salt content of the film;

Although the comparison was made between PEMs in Milli-Q water environment, a portion of the $\mathrm{Na}^{+}$and $\mathrm{Cl}^{-}$ions absorbed by the PEM during the contact with the saline solutions could not diffuse out during the rinsing and there are slight differences in internal salinity of the film. This results in 
the increased swelling and increased water content of the PEM. Detachment of the polyelectrolytes from the PEM (mechanism I) results in an increase in the frequency, whereas higher water content of the film results in a reduction in the frequency. As such I and II can act against each other. The final observed frequency is a summation of effects from I and II.

\subsubsection{Negative capping layer}

PEMs formed in four layers are ending in PSS and thus possess a negative surface charge. A summary of results for these PEMs is available in Fig. 6. Considering the sensitivity of the analysis and the error values, It can be claimed that the PEMs formed from unmodified PEI fully retained their stability until the salinity of $4.75 \mathrm{M}$. A more or less stable film results in small absolute values for mechanism I which are comparable to absolute values of frequency shift due to II. Thus the summation of I and II results in small changes resonating around the zero value. The situation was more complex for the case of the modified PEI/PSS film. An initial mass loss was observed on the first contact with saline medium, whereby up to $20 \%$ of the PEM mass was lost. However, the multilayer becomes more stable after this initial loss and retains most of its weight until reaching salinity of $4.75 \mathrm{M}$ $\mathrm{NaCl}$.

\subsubsection{Positive capping layer}

Five layered PEMs end in polycation and possess positive surface charge. Results from this set of experiments are reflected in Fig. 7. Here, in both cases of modified and unmodified polycations we observe less stable films. In the case of unmodified PEI, an initial mass loss of around $25 \%$ is observed. This loss increases to up to $60 \%$ by reaching $1.5 \mathrm{M}$ and up to $75 \%$ by reaching 
1.75M. Further increase in the salinity doesn't result in considerable mass loss (refer to Fig. 7). The 5 layer PE films are clearly unstable and extremely susceptible to salinity. Furthermore, the stepwise loss in the mass suggests removal of the layers from the film and conforms with the quasi layered (versus completely mixed) structure.

To best explain the observed behaviors, it's helpful to consider the "in and out" mechanism of LBL deposition as articulated by Richert et al. [55] and discussed in [56]. Observed results can be explained by the relative mobility of the three PEs involved. Polymer mobility is a complex function of the rigidity, size, pH, linearity, etc. [56]. However, it is known that polymer diffusion is roughly inversely proportional to its molecular weight[57]. The huge difference in the molecular weight of the PEI and PSS used in this study insures lower mobility of the polycation. This is further reinforced with highly branched structure of the PEI. Modified PEIs, possess relatively spacious crownether pendants that reduce their mobility even further. Moreover, crownethers can form hydrogen bonds with protonated amines [15] which can act as weak cross linking in PEI chains and further reduce the mobility of the modified polycation and hinder PSS diffusion into them. Consequently, the order of mobility of PEs would be PSS $\gg \mathrm{PEI}>$ modified PEI. As such, we expect low degree of intermixing between layers in the case of both modified and unmodified PEMs, with the modified PEMs being even less intermixed than their unmodified counterparts. The results of the experiment (linear growth, refer to section 3.1) conform with this expectations as well. Moreover, this explanation is in agreement with the molecular dynamics simulation and experimental results of Lee et al. [58] who found PEM films made from bigger polymers more layered and suggested a similar explanation for it. 
As mentioned by the [56], in these situations the more mobile polymer diffuses in the film while the less mobile species retains its more or less layered structure. We hypothesize that the PSS chains are diffusing inside the previously deposited layers and by complexing with PEI act like a cross linker between previous PE chains. In the absence of final capping PSS layer, the links between top poly cation chains $\left(5^{\text {th }}\right.$ layer $)$ and rest of the film is exceptionally weak. This effect is more pronounced in the case of modified PEIs and explains the less stable PEMs with positive capping layer.

Once the inadequately anchored $5^{\text {th }}$ layer is removed, an interesting case arises from comparing with the 4-layered membranes. It appears that upon addition of the less mobile PEI layer, stability of the previous layers is also compromised. However this is expected in lights of the "in and out" model. Upon addition of the PEI layer, PSS chains present in the lower layers diffuse towards the surface, weakening the internal links of the first four layers.

Differences observed between the modified and unmodified cases of 4layered PEMs can be explained in a similar fashion. Lower mobility of the modified PEI reduces the relative mobility of the polymer chains and hinders PSS. This reduces the stability, but is not as severe as the case of five layered membranes and once the top layers lose their inadequately anchored polyelectrolytes, the PEM becomes stable.

In both modified and unmodified PEMs, a complete dissolution of the PEM structure was not achievable by increasing salinity and a $2 \%$ solution of Hellmanex ${ }^{\circledR}$ III solution (Hellma GmbH, Germany) providing a pH of 12 had to be used to completely remove the adsorbed films. 


\subsection{Filtration experiments}

Salt rejection experiments were performed with both types of membranes and selectivity between different cations was followed as

$$
\alpha_{\mathrm{Li} / \mathrm{K}}=\frac{\frac{C_{\mathrm{Li}, \text { permeate }}}{C_{\mathrm{Li}, \text { feed }}}}{\frac{C_{\mathrm{K}, \text { permeate }}}{C_{\mathrm{K}, \text { feed }}}}
$$

Wherein $\mathrm{C}_{\mathrm{x}}$ denotes concentration of species $\mathrm{x}$ in ppm. The permeability of the membrane towards water and different solutes was followed by water and solute permeability (A and B) constants in Solution-Diffusion model [59]. Corrections for the concentration polarization phenomena were made based on the Film theory [60].

$$
\begin{gathered}
J_{\mathrm{w}}=A(\Delta P-\Delta \pi) \\
\frac{C_{\mathrm{m}}-C_{\mathrm{p}}}{C_{\mathrm{b}}-C_{\mathrm{p}}}=\frac{\frac{R_{\text {real }}}{1-R_{\text {real }}}}{\frac{R_{\mathrm{obs}}}{1-R_{\mathrm{obs}}}}=\exp \left(\frac{J_{\mathrm{w}}}{k}\right) \\
B=\frac{J_{\mathrm{s}}}{C_{\mathrm{m}}-C_{\mathrm{p}}}=\frac{J_{\mathrm{w}}\left(1-R_{\mathrm{obs}}\right)}{R_{\mathrm{obs}} \exp \left(\frac{J_{\mathrm{w}}}{k}\right)}
\end{gathered}
$$

Wherein $J_{\mathrm{w}}$ and $J_{\mathrm{s}}$ denote water and solute flux. $\Delta P$ and $\Delta \pi$ represent applied pressure difference and osmotic pressure difference. $C_{\mathrm{p}}, C_{\mathrm{b}}$ and $C_{\mathrm{m}}$ stand for concentration of the solute at the permeate, feed and membrane surface respectively. And finally $R_{\text {obs }}$ and $R_{\text {real }}$ denote observed and real rejections. The mass transfer coefficient, $\mathrm{k}$, was calculated as: [61]

$$
\begin{gathered}
S h=k r / D=0.023 R e^{0.567} S c^{0.33} \\
R e=\omega r^{2} / \nu
\end{gathered}
$$

Wherein Sh, Re and Sc denote Sherwood, Reynolds and Schmidt numbers and $\omega$ and $r$ represent the angular velocity and radius of the stirrer . The 
diffusion coefficient, D, was calculated for the feed concentration of the salts according to the equations provided by Lobo [62].

\subsubsection{Behavior in the transient state}

In experiments with modified membranes performance of the membrane in terms of solute rejection was a function of time, allowing for two stages to be differentiated. At the start of the experiment a transient step could be identified, represented by the gray zone in Fig.8. After about 90 minutes, a plateau was reached and the performance of the membrane was stabilized. In the case of membranes prepared from unmodified PEI, the value of $\alpha_{\mathrm{Li} / \mathrm{K}}$ was constant and close to that of the feed. Therefore, the quasi breakthrough curve observed in the transient step can be associated with the complexation of cations and crown ether moieties. We postulate that at around 90 minutes, all crownether sites are saturated and selectivity of the membrane is lost. A similar behavior and explanation is also reported by Tas et al. in crownether containing ion-exchange membranes [42]. It is worth mentioning that regenerated membranes exhibit comparable behavior over several regeneration cycles and as such, the loss of selectivity can not be associated with the instability of the membranes.

The modified membranes show a tendency towards hindering the lithium more than potassium during the transient step, reflected in $\alpha_{\mathrm{Li} / \mathrm{K}}$ values of lower than one. This points to a stronger interaction between lithium ions and the crown ether in comparison to the potassium cations. Although the order of preference of free crown ether in the bulk is in reverse (refer to Table 1), a change in the complexation was expected in crown ethers inside the membrane pores. Potassium is generally believed to form sandwich type 2:1 complexes with the 15-crown-5 [23]. A complex type that is severely 


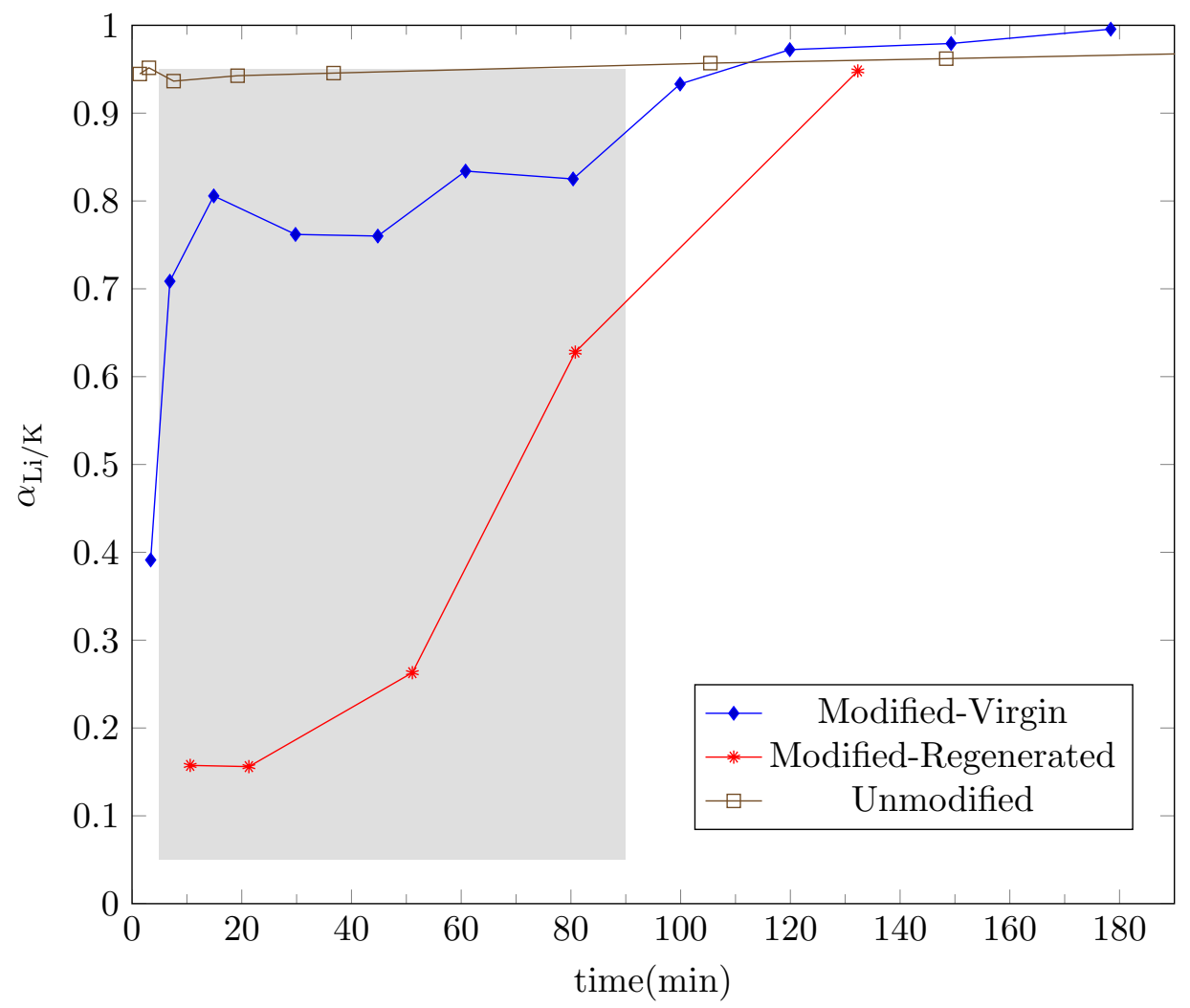

Figure 8: $\alpha_{\mathrm{Li} / \mathrm{K}}$ as a function of time

hampered due to the steric hindrance in highly branched structure of the PEI. In contrast, lithium ions are smaller and form 1:1 complexes. We hypothesize that the effect of steric hindrance has reduced the possibility of 2:1 complex formation for potassium, leading to stronger lithium complexes in comparison to potassium ones.

It is worth mentioning that each crownether molecule has been in contact with several cations before it reaches the saturation point. By rough estimations, close to $13 \mathrm{nmol}$ of crownether are present in membrane coupons (refer to the supporting materials for detailed calculation of this value). In 


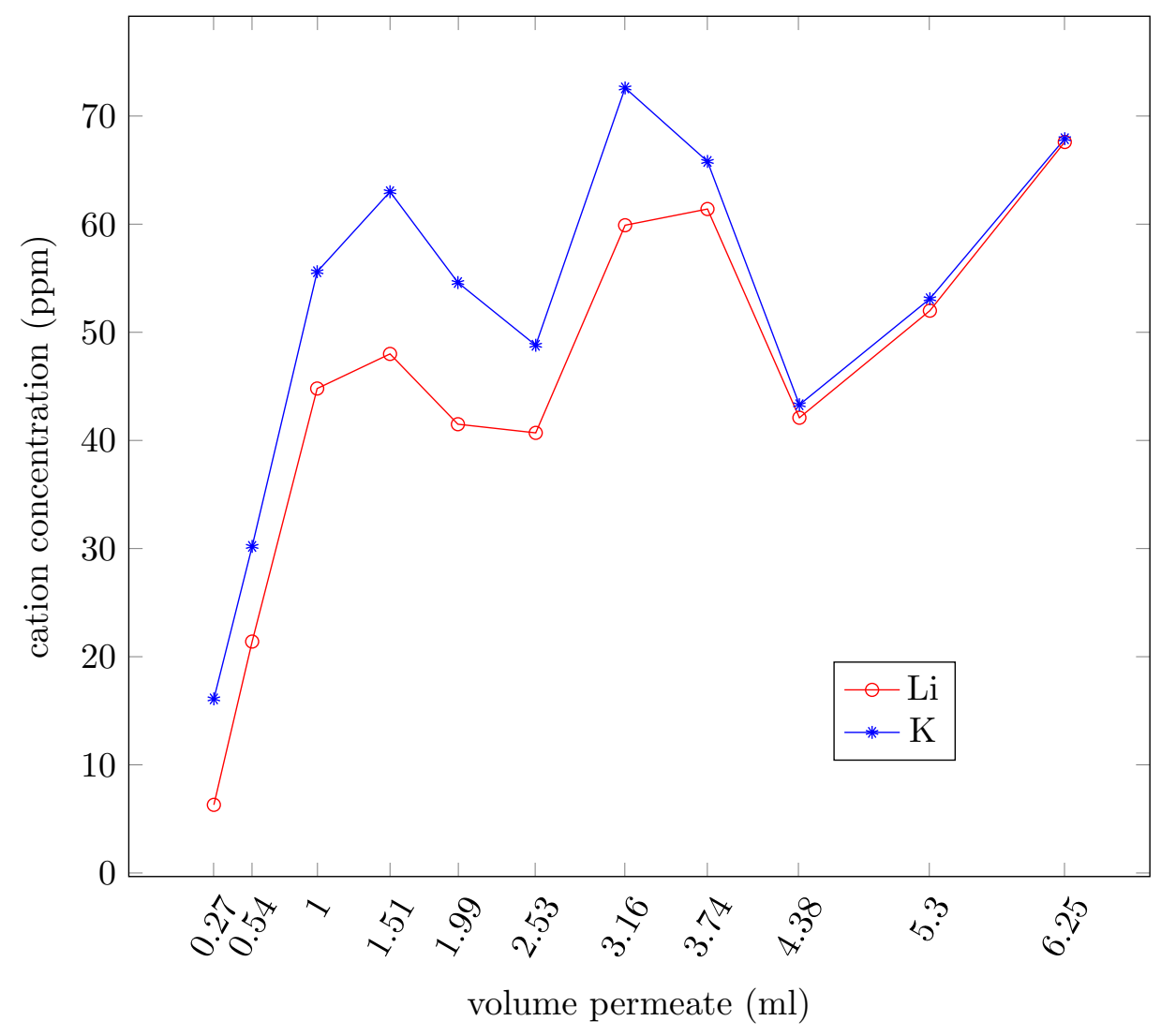

Figure 9: Cation concentration in the permeate as a function of the volume of the permeate. Experiment performed on virgin modified membrane and mixed salt conditions.

a typical sample of regenerated membranes in mixed salt feed, about 11 $\mu$ mol lithium and $5 \mu \mathrm{mol}$ potassium have passed through the membrane before reaching the 90 minute mark. As a result, it can be expected that each crown ether molecule undergoes several complexation/decomplexation cycles before reaching the saturation point. This suggests a kinetic factor that limits the release of the complexed ions, leading to the eventual saturation and exhaustion of all crownether sites. However the exact reason and mechanism behind the saturation of complexing sites remains elusive and 
further investigations are needed to validate this claim.

\subsubsection{Behavior in steady state}

In general, adding the crownether molecules to the PEI chain resulted in membranes with lowered permeability and increased salt rejection. Upon PEI modification, water permeability constant (A) was lowered by close to $70 \%$ from 4.53 E-7 to 1.26 E-7 $\frac{m}{s \text { bar }}$. At the same time, the salt rejection of the membranes increased from a value of $6.8 \%$ to $71.6 \%$ for the case of $\mathrm{KCl}$ and $3.5 \%$ to $41.1 \%$ in the case of $\mathrm{LiCl}$ in crownether containing coupons. This is in accordance with the changes as big as one order of magnitude in the solute permeability (B) constants (refer to table 3 ). While such a behavior is in agreement with the general permeability/selectivity trade-off [63], its nature should be further investigated. One of the possible explanations can be the increase in the thickness of the modified membranes. In order to verify this hypothesis, a comparison between 6 layer (3 bilayer) and 14 layer (7 bilayer) membranes was made (for a detailed explanation of the synthesis of 14 layer membranes and the performed experiment please refer to the supporting information). While the unmodified 14 layer membrane is thicker than its 6 layer modified counterpart, it still shows a lower rejection. Therefore, increased thickness can not be assumed the sole reason for the increased rejections.

Since the lack of cation selectivity in this step rules out any effect from complexation, we postulate that a structural difference between the two PEMMs is responsible for the increased rejection. The structural difference can be caused by the change in mobility and hydrophobicity of the PEI upon modification and is also suggested by the QCM-D results of Sec. 2.2 and Sec. 3.2. For example, a more layered structure is expected to increase 
Table 3: Water and solute permeability constants A and B for single salt experiments at steady state.

\begin{tabular}{c|c|c|c} 
Membrane & $\mathbf{A}\left(\frac{m}{s b a r}\right)$ & $\mathbf{B}_{\mathbf{L i}}(\mathrm{m} / \mathrm{s})$ & $\mathbf{B}_{\mathbf{K}}(\mathrm{m} / \mathrm{s})$ \\
\hline & \multicolumn{3}{|c}{} \\
Unmodified PEI/PSS & $4.53 \pm 0.49 \mathrm{E}-7$ & $7.0 \pm 0.03 \mathrm{E}-8$ & $6.8 \pm 2.4 \mathrm{E}-8$ \\
modified PEI/PSS & $1.26 \pm 0.08 \mathrm{E}-7$ & $1.4 \pm 0.1 \mathrm{E}-8$ & $9 \pm 2 \mathrm{E}-9$
\end{tabular}

574 rejection. This will be the subject of a future study. It is worth mentioning

that at steady state, the solute permeability factor (B) of lithium is 1.5 times higher that of one of the potassium. However, this is not in contradiction to the results of the transient state ( $\alpha$ values). The crown ether sites are all saturated at this stage and do not affect the ion transportation. The higher permeability of lithium in this stage can be contributed to its higher intrinsic mobility in the membrane.

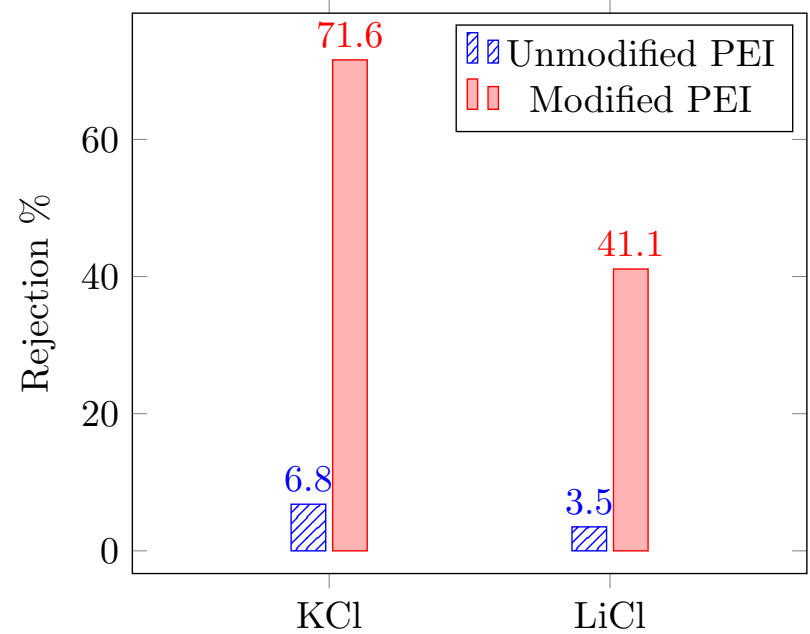

Figure 10: Comparison of Rejection values for $\mathrm{Li}$ and $\mathrm{K}$ in single salt experiments at steady state step. 


\subsection{Applicability}

The goal of this study was to achieve a lithium reach permeate stream by manufacturing a monovalent selective PEMM membrane. However, the resulted membranes exhibited a potassium selective nature. Nevertheless, we believe the results of the study can contribute to the goal of the study as a proof of concept.

Cation selectivity of the PEMM membranes with embedded crown-ether moieties is proven in this study. The authors postulate that replacing the chosen crown-ether with other ligands of this family results in membranes with different cation selectivities. It should be noted that the synthesis route of the crown ether moiety (section 2.1.1) is not exclusive to the 15crown-5. Different crown ethers can be synthesized via the same route by changing the linear ether precursor (here bis[2-(2-chloroethoxy)ethyl]ether). The presented synthesis procedure can thus be used for other cation/crown ether pairs as well. Selection of the appropriate crownether for the future studies can be based on the reasoning provided in this manuscript.

\section{Conclusion}

The crown ether molecule 15 -crown-5 was synthesized and incorporated in the PEI chains. The modified PEI polymers were used as one of the poly electrolytes in LBL deposition in conjugation with PSS as poly anion. The stability of the PEM was established on silica substrate and the PEMMs were prepared and characterized in terms of water, lithium and potassium permeability. The synthesized membranes showed selectivity only for a defined period of time. 


\section{Acknowledgment}

This project has received funding from European Union's Horizon 2020 research and innovation program under Marie Skłodowska-Curie grant agreement $N^{\circ} 676070$. Authors would like to thank Ir. Lorenz De Neve for assisting in NMR analysis, Ir. Christophe vandeputte who synthesized modified polymers as his master thesis and Ir. Pieter Naert for his invaluable inputs on the synthesis of the polymers and assistance with the HNMR analysis.

\section{References}

[1] Sixie Yang, Fan Zhang, Huaiping Ding, Ping He, and Haoshen Zhou. Lithium Metal Extraction from Seawater, sep 2018. ISSN 25424351. URL https://www.sciencedirect.com/science/ article/pii/S2542435118302927.

[2] Rey Eliseo C. Torrejos, Grace M. Nisola, Myoung Jun Park, Ho Kyong Shon, Jeong Gil Seo, Sangho Koo, and Wook Jin Chung. Synthesis and characterization of multi-walled carbon nanotubes-supported dibenzo-14-crown-4 ether with proton ionizable carboxyl sidearm as Li+adsorbents. Chem. Eng. J., 264:89-98, 2015. ISSN 13858947. doi: 10.1016/j.cej.2014.11.036.

[3] Mamadou S. Diallo, Madhusudhana Rao Kotte, and Manki Cho. Mining Critical Metals and Elements from Seawater: Opportunities and Challenges. Environ. Sci. Technol., 49(16):9390-9399, 2015. ISSN 15205851. doi: 10.1021/acs.est.5b00463.

[4] Pratima Meshram, B D Pandey, and T R Mankhand. Extraction of lithium from primary and secondary sources by pre-treatment, leaching 
and separation: A comprehensive review, 2014. ISSN 0304386X. URL http://dx.doi.org/10.1016/j.hydromet.2014.10.012.

[5] Kazuharu Yoshizuka, Marek Holba, Takeshi Yasunaga, and Yasuyuki Ikegami. Performance Evaluation of Benchmark Plant for Selective Lithium Recovery from Seawater. J. Ion Exch., 18(4):450-453, oct 2007. ISSN 0915-860X. doi: 10.5182/jaie.18.450. URL http://joi.jlc.jst. go . jp/JST . Journalarchive/jaie1990/18 .450?from=CrossRef.

[6] Reuters news agency. South Korea aims to produce lithium from seawater, 2011. https://www.reuters.com/article/ us-korea-lithium-idUSTRE70J3GG20110120 [Accessed: March 10th-2019].

[7] Ayuko Kitajou, Takuya Suzuki, Syouhei Nishihama, and Kazuharu Yoshizuka. Selective recovery of lithium from seawater using a novel $\mathrm{MnO} 2$ type adsorbent II - Enhancement of lithium ion selectivity of the adsorbent. Ars Separatoria Acta, 2:97-106, 2003.

[8] Katsuhiko Ariga, Jonathan P. Hill, and Qingmin Ji. Layer-by-layer assembly as a versatile bottom-up nanofabrication technique for exploratory research and realistic application. Phys. Chem. Chem. Phys., 9(19):2319, 2007. ISSN 1463-9076. doi: 10.1039/b700410a. URL http://xlink.rsc.org/?DOI=b700410a.

[9] Joris de Grooth, Radek Oborný, Jens Potreck, Kitty Nijmeijer, and Wiebe M. de Vos. The role of ionic strength and odd-even effects on the properties of polyelectrolyte multilayer nanofiltration membranes. $J$. Memb. Sci., 475:311-319, 2015. ISSN 18733123. doi: 10.1016/j.memsci. 
2014.10.044. URL http://dx.doi.org/10.1016/j.memsci.2014.10. 044.

[10] Oishi Sanyal and Ilsoon Lee. Recent Progress in the Applications of Layer-By-Layer Assembly to the Preparation of Nanostructured Ion-Rejecting Water Purification Membranes. J. Nanosci. Nanotechnol., 14(3):2178-2189, 2014. ISSN 1533-4880. doi: 10.1166/ jnn.2014.8541. URL http://www. ingentaconnect.com/content/10. $1166 / j n n .2014 .8541$.

[11] Nithya Joseph, Pejman Ahmadiannamini, Richard Hoogenboom, and Ivo F.J. Vankelecom. Layer-by-layer preparation of polyelectrolyte multilayer membranes for separation, feb 2014. ISSN 17599962. URL http://xlink.rsc.org/?DOI=C3PY01262J.

[12] Guo Rong Xu, Sheng Hui Wang, He Li Zhao, Shui Bo Wu, Jian Mei $\mathrm{Xu}, \mathrm{Lu} \mathrm{Li}$, and Xiao Yu Liu. Layer-by-layer (LBL) assembly technology as promising strategy for tailoring pressure-driven desalination membranes. J. Memb. Sci., 493:428-443, 2015. ISSN 18733123. doi: 10.1016/j.memsci.2015.06.038.

[13] C. J. Pedersen. Cyclic Polyethers and Their Complexes with Metal Salts. J. Am. Chem. Soc., 89(26):7017-7036, 1967. ISSN 15205126. doi: $10.1021 /$ ja01002a035.

[14] N. Kent Dalley. STRUCTURAL STUDIES OF SYNTHETIC MACROCYCLIC MOLECULES AND THEIR CATION COMPLEXES. Synth. Multident. Macrocycl. Compd., pages 207-243, jan 1978. doi: 10.1016/ B978-0-12-377650-1.50009-5. URL https://www.sciencedirect. com/science/article/pii/B9780123776501500095. 
[15] F. De Jong and D N Reinhoudt. Stability and Reactivity of CrownEther Complexes. Adv. Phys. Org. Chem., 17(C):279-433, 1980. ISSN 00653160. doi: 10.1016/S0065-3160(08)60130-6.

[16] Sanjib Senapati and Amalendu Chandra. Dielectric constant of water confined in a nanocavity. J. Phys. Chem. B, 105(22):5106-5109, 2001. ISSN 10895647. doi: 10.1021/jp011058i.

[17] Yingchun Liu, Qi Wang, and Linghong Lu. Transport Properties and Distribution of Water Molecules Confined in Hydrophobic Nanopores and Nanoslits. (22):6921-6926, 2004.

[18] Berrin Tansel. Significance of thermodynamic and physical characteristics on permeation of ions during membrane separation: Hydrated radius, hydration free energy and viscous effects. Sep. Purif. Technol., 86:119-126, 2012. ISSN 13835866. doi: 10.1016/j.seppur.2011.10.033. URL http://dx.doi.org/10.1016/j.seppur.2011.10.033.

[19] Rey Eliseo C. Torrejos, Grace M. Nisola, Ho Seong Song, Lawrence A. Limjuco, Chosel P. Lawagon, Khino J. Parohinog, Sangho Koo, Jeong Woo Han, and Wook Jin Chung. Design of lithium selective crown ethers: Synthesis, extraction and theoretical binding studies. Chem. Eng. J., 326:921-933, 2017. ISSN 13858947. doi: 10.1016/j.cej. 2017.06.005. URL http://dx.doi.org/10.1016/j.cej .2017.06.005.

[20] Yosuke Ando, Yuki Hiruta, Daniel Citterio, and Koji Suzuki. A highly Li+-selective glass optode based on fluorescence ratiometry. Analyst, 134(11):2314-2319, oct 2009. ISSN 00032654. doi: 10.1039/b912756a. URL http://xlink.rsc.org/?DOI=b912756a. 
[21] Roger C. Helgeson, Joseph M. Timko, and Donald J. Cram. Structural requirements for cyclic ethers to complex and lipophilize metal cations or .alpha.-amino acids. J. Am. Chem. Soc., 95(9):3023-3025, may 1973. ISSN 0002-7863. doi: 10.1021/ja00790a053. URL http://pubs.acs . org/doi/abs/10.1021/ja00790a053.

[22] Reed M. Izatt, Jerald S. Bradshaw, Steven A. Nielsen, John D. Lamb, James J. Christensen, and Debabrata Sen. Thermodynamic and Kinetic Data for Cation-Macrocycle Interaction. Chem. Rev., 85(4):271-339, 1985. ISSN 15206890. doi: 10.1021/cr00068a003.

[23] P. R. Mallinson and Mary R. Truter. Crystal structures of complexes between alkali-metal salts and cyclic polyethers. Part V. The 1 : 2 complex formed between potassium iodide and $2,3,5,6,8,9,11,12$ octahydro-1,4,7,10,13-benzopentaoxacyclopentadecin (benzo-15-crown5). J. Chem. Soc. Perkin Trans. 2, 0(12):1818, jan 1972. ISSN 03009580. doi: 10.1039/P29720001818. URL http://xlink.rsc.org/ ?DOI=p29720001818.

[24] Rey Eliseo C. Torrejos, Grace M. Nisola, Ho Seong Song, Jeong Woo Han, Chosel P. Lawagon, Jeong Gil Seo, Sangho Koo, Hern Kim, and Wook-Jin Chung. Liquid-liquid extraction of lithium using lipophilic dibenzo-14-crown-4 ether carboxylic acid in hydrophobic room temperature ionic liquid. Hydrometallurgy, 164:362-371, sep 2016. ISSN 0304-386X. doi: 10.1016/J.HYDROMET.2016.05.010. URL https://www.sciencedirect.com/science/article/abs/pii/ S0304386X16302754.

[25] Kazuya Kobiro. New class of lithium ion selective crown ethers 
with bulky decalin subunits. Coord. Chem. Rev., 148:135-149, feb 1996. ISSN 0010-8545. doi: 10.1016/0010-8545(96)01209-X. URL https://www.sciencedirect.com/science/article/abs/pii/ $001085459601209 X$.

[26] Koji. Suzuki, Hiroyuki. Yamada, Kazunari. Sato, Kazuhiko. Watanabe, Hideaki. Hisamoto, Yoshito. Tobe, and Kazuya. Kobiro. Design and synthesis of highly selective ionophores for lithium ion based on 14crown-4 derivatives for an ion-selective electrode. Anal. Chem., 65(23): 3404-3410, dec 1993. ISSN 0003-2700. doi: 10.1021/ac00071a012. URL http://pubs.acs.org/doi/abs/10.1021/ac00071a012.

[27] Keiichi Kimura, Takumi Maeda, and Toshiyuki Shono. New Poly - and Bis(crown ether)s as Extracting Reagents. Anal. Lett., 11(10):821-827, jan 1978. ISSN 0003-2719. doi: 10.1080/ 00032717808059734. URL http://www.tandfonline.com/doi/abs/ $10.1080 / 00032717808059734$.

[28] Basudev Swain. Separation and purification of lithium by solvent extraction and supported liquid membrane, analysis of their mechanism: a review. J. Chem. Technol. Biotechnol., 91(10):2549-2562, 2016. ISSN 10974660. doi: 10.1002/jctb.4976.

[29] Roya Mohammad Zadeh Kakhki and Gholamhossein Rounaghi. Competitive Bulk Liquid Membrane Transport of Heavy Metal Cations Using the 18-Crown-6 Ligand as an Ionophore. J. Chem. Eng. Data, 56(7):3169-3174, jul 2011. doi: 10.1021/je200220d. URL http: //pubs.acs.org/doi/abs/10.1021/je200220d.

[30] W. S. Winston Ho, Bing Wang, Travis E. Neumuller, and Justin Roller. 
Supported liquid membranes for removal and recovery of metals from waste waters and process streams. Environ. Prog., 20(2):117-121, 2001. ISSN 02784491. doi: 10.1002/ep.670200215.

[31] Deneikah T. Jackson and Peter N. Nelson. Preparation and properties of some ion selective membranes: A review. J. Mol. Struct., 1182:241259, apr 2019. ISSN 00222860. doi: 10.1016/j.molstruc.2019.01.050. URL https://www.sciencedirect.com/science/article/pii/ S0022286019300602https://linkinghub.elsevier.com/retrieve/ $\mathrm{pii/S0022286019300602.}$

[32] Lawrence A. Limjuco, Grace M. Nisola, Rey Eliseo C. Torrejos, Jeong Woo Han, Ho Seong Song, Khino J. Parohinog, Sangho Koo, Seong Poong Lee, and Wook Jin Chung. Aerosol Cross-Linked Crown Ether Diols Melded with Poly(vinyl alcohol) as Specialized Microfibrous Li+Adsorbents. ACS Appl. Mater. Interfaces, 9(49):4286242874, dec 2017. ISSN 19448252. doi: 10.1021/acsami.7b14858. URL http://pubs.acs.org/doi/10.1021/acsami.7b14858.

[33] Lynn M. Dulyea, Thomas M. Fyles, and G. David Robertson. Coupled transport membranes incorporating a polymeric crown ether carboxylic acid. J. Memb. Sci., 34(1):87-108, 1987. ISSN 03767388. doi: 10.1016/ S0376-7388(00)80022-8.

[34] A. Gherrou, H. Kerdjoudj, R. Molinari, P. Seta, and E. Drioli. Fixed sites plasticized cellulose triacetate membranes containing crown ethers for silver(I), copper(II) and gold(III) ions transport. J. Memb. Sci., 228 (2):149-157, 2004. ISSN 03767388. doi: 10.1016/j.memsci.2003.10.003.

[35] Abdelaziz Gherrou, Hacène Kerdjoudj, Raffaele Molinari, and Patrick 
Seta. Preparation and characterization of polymeric plasticized membranes (PPM) embedding a crown ether carrier application to copper ions transport. Mater. Sci. Eng. C, 25(4):436-443, 2005. ISSN 09284931. doi: 10.1016/j.msec.2004.11.002.

[36] P. K. Mohapatra, D. S. Lakshmi, A. Bhattacharyya, and V. K. Manchanda. Evaluation of polymer inclusion membranes containing crown ethers for selective cesium separation from nuclear waste solution. J. Hazard. Mater., 169(1-3):472-479, 2009. ISSN 03043894. doi: 10.1016/j.jhazmat.2009.03.124.

[37] Adam J. Schow, Randall T. Peterson, and John D. Lamb. Polymer inclucion membranes containing marocyclic carriers for use in cation separations. J. Memb. Sci., 111:291-295, 1996. ISSN 03767388. doi: 10.1016/0376-7388(95)00295-2.

[38] Takashi. Hayashita and Richard A. Bartsch. Competitive Sorption of Alkali-Metal and Alkaline-Earth-Metal Cations by Carboxylic Acid Resins Containing Acyclic or Cyclic Polyether Units. Anal. Chem., 63 (17):1847-1850, nov 1991. ISSN 15206882. doi: 10.1021/ac00017a033. URL http://pubs.acs.org/doi/abs/10.1021/ac00220a006.

[39] Qiang Zhao and Richard A. Bartsch. Synthesis of formaldehydedibenzo-18-crown-6 condensation resins containing phosphonic acid groups and their alkali-metal cation sorption. J. Appl. Polym. Sci., 57(12):1465-1471, sep 1995. ISSN 10974628. doi: 10.1002/app. 1995.070571206. URL http://doi.wiley.com/10.1002/app.1995. 070571206.

[40] E. Elizabeth Laney, Joung Hae Lee, Jong Seung Kim, Xiaowu Huang, 
Youngchan Jang, Hong Sik Hwang, Takashi Hayashita, and Richard A. Bartsch. Sorption of lead(II) by proton-ionizable polyether resins. React. Funct. Polym., 36(2):125-134, mar 1998. ISSN 13815148. doi: 10.1016/S1381-5148(97)00108-9. URL https://www. sciencedirect. com/science/article/abs/pii/S1381514897001089.

[41] Alain Favre-Réguillon, Nathalie Dumont, Branko Dunjic, and Marc Lemaire. Polymeric and immobilized crown compounds, material for ion separation. Tetrahedron, 53(4):1343-1360, jan 1997. ISSN 0040-4020. doi: 10.1016/S0040-4020(96)01072-1. URL https://www . sciencedirect.com/science/article/pii/S0040402096010721.

[42] Sinem Tas, Bram Zoetebier, Mark A. Hempenius, G. Julius Vancso, and Kitty Nijmeijer. Monovalent cation selective crown ether containing poly(arylene ether ketone)/SPEEK blend membranes. $R S C A d v$, 6 (60):55635-55642, jun 2016. ISSN 20462069. doi: 10.1039/c6ra11566g. URL http://xlink.rsc.org/?DOI=C6RA11566G.

[43] Spiro D. Alexandratos and Christy L. Stine. Synthesis of ion-selective polymer-supported crown ethers: A review. React. Funct. Polym., 60 (1-3):3-16, 2004. ISSN 13815148. doi: 10.1016/j.reactfunctpolym.2004. 02.006 .

[44] Alfred J. Smetana and Alexander I. Popov. Lithium-7 nuclear magnetic resonance and calorimetric study of lithium crown complexes in various solvents. J. Solution Chem., 9(3):183-196, mar 1980. ISSN 00959782. doi: 10.1007/BF00648325. URL http://link.springer. com/10.1007/BF00648325.

[45] R. M. Izatt, R. E. Terry, B. L. Haymore, L. D. Hansen, N. K. Dailey, 
A. G. Ayondet, and J. J. Christensen. Calorimetric Titration Study of the Interaction of Several Uni-and Bivalent Cations with 15-Crown-5, 18-Crown-6, and Two Isomers of Dicyclohexo-18-Crown-6 in Aqueous Solution at $25^{\circ} \mathrm{c}$ and $\mu=0.1$. J. Am. Chem. Soc., 98(24):7620-7626, nov 1976. ISSN 15205126. doi: 10.1021/ja00440a028. URL http: //pubs.acs.org/doi/abs/10.1021/ja00440a028.

[46] R. M. Izatt, R. E. Terry, D. P. Nelson, Y. Chan, D. J. Eatough, J. S. Bradshaw, L. D. Hansen, and J. J. Christensen. Calorimetric Titration Study of the Interaction of Some Uni-and Bivalent Cations with Benzo-15-Crown-5, 18-Crown-6, Dibenzo-24-Crown-8, and Dibenzo-27Crown-9 in Methanol-Water Solvents, at $25^{\circ} \mathrm{C}$ and $\mu=0.1$. J. Am. Chem. Soc., 98(24):7626-7630, nov 1976. ISSN 15205126. doi: 10. 1021/ja00440a029. URL http://pubs.acs.org/doi/abs/10.1021/ ja00440a029.

[47] Jy Dale Lin and Alexander I. Popov. Nuclear Magnetic Resonance Studies of Some Sodium Ion Complexes with Crown Ethers and [2] Cryptands in Various Solvents. J. Am. Chem. Soc., 103(13):37733777, jul 1981. ISSN 15205126. doi: 10.1021/ja00403a026. URL http://pubs.acs.org/doi/abs/10.1021/ja00403a026.

[48] Chadzynsky Henryk Cygan Andrzej, Biernat Jan F. Macrocyclic Polyfunctional Lewis Bases . Part III . Electrophoretic Behavior of Macrocyclic Polyethers. Pol. J. Chem., 53(January 1979):929-933, 1979.

[49] R. Ungaro, B. El Haj, and J. Smid. Substituent Effects on the Stability of Cation Complexes of 4-Substituted Monobenzo Crown Ethers. J. 
Am. Chem. Soc., 98(17):5198-5202, 1976. ISSN 15205126. doi: 10. 1021/ja00433a024.

[50] Artur Kasprzak, Magdalena Popławska, Michał Bystrzejewski, Olga Łabȩdź, and Ireneusz P. Grudziński. Conjugation of polyethylenimine and its derivatives to carbon-encapsulated iron nanoparticles. $R S C$ Adv., 5(104):85556-85567, 2015. ISSN 2046-2069. doi: 10.1039/ C5RA17912B. URL http://xlink.rsc.org/?DOI=C5RA17912B.

[51] Akira Baba, Futao Kaneko, and Rigoberto C Advincula. Polyelectrolyte adsorption processes characterized in situ using the quartz crystal microbalance technique : alternate adsorption properties in ultrathin polymer films. 173:39-49, 2000.

[52] Sinem Tas, Ozge Kaynan, Elif Ozden-Yenigun, and Kitty Nijmeijer. Polyacrylonitrile (PAN)/crown ether composite nanofibers for the selective adsorption of cations. $R S C A d v ., 6(5): 3608-3616$, jan 2016. ISSN 20462069. doi: 10.1039/c5ra23214g. URL http://xlink.rsc.org/ ?DOI=C5RA23214G.

[53] Nazish Hoda and Ronald G. Larson. Modeling the buildup of exponentially growing polyelectrolyte multilayer films. J. Phys. Chem. B, 113 (13):4232-4241, 2009. ISSN 15206106. doi: 10.1021/jp809959j.

[54] Ilya Reviakine, Diethelm Johannsmann, and Ralf P. Richter. Hearing what you cannot see and visualizing what you hear: Interpreting quartz crystal microbalance data from solvated interfaces. Anal. Chem., 83 (23):8838-8848, 2011. ISSN 00032700. doi: 10.1021/ac201778h.

[55] Ludovic Richert, Philippe Lavalle, Elisabeth Payan, Xiao Zheng Shu, 
Glenn D Prestwich, Jean-François Stoltz, Pierre Schaaf, Jean-claude Voegel, and Catherine Picart. Layer by Layer Buildup of Polysaccharide Films: Physical Chemistry and Cellular Adhesion Aspects. Langmuir, 20(2):448-458, jan 2004. ISSN 0743-7463. doi: 10.1021/la035415n. URL https://pubs.acs.org/doi/10.1021/la035415n.

[56] Hadi M. Fares and Joseph B. Schlenoff. Diffusion of Sites versus Polymers in Polyelectrolyte Complexes and Multilayers. J. Am. Chem. Soc., 139(41):14656-14667, oct 2017. ISSN 0002-7863. doi: 10.1021/jacs. 7b07905. URL http://pubs.acs.org/doi/10.1021/jacs.7b07905.

[57] R. A. Pethrick. The theory of polymer dynamics M. Doi and S. F. Edwards, Oxford University Press, Oxford, 1986. pp. xiii +391 , price $£ 40.00$. ISBN 0-19-85 1976-1. Br. Polym. J., 20(3):299-299, 1988. ISSN 00071641. doi: 10.1002/pi.4980200324. URL http://doi.wiley.com/ $10.1002 / \mathrm{pi} .4980200324$.

[58] Hwankyu Lee. Effect of polyelectrolyte size on multilayer conformation and dynamics at different temperatures and salt concentrations. $J$. Mol. Graph. Model., 70:246-252, 2016. ISSN 18734243. doi: 10.1016/j. jmgm.2016.09.014. URL http://dx.doi.org/10.1016/j.jmgm. 2016. 09.014.

[59] J.GBaker Wijmans and R.W. Baker. The solution-diffusion model: a review. J. Memb. Sci., 107(1-2):1-21, nov 1995. ISSN 03767388. doi: 10.1016/0376-7388(95)00102-I. URL https ://linkinghub.elsevier. com/retrieve/pii/037673889500102I.

[60] Marcel Mulder. Basic Principles of Membrane Technology. Springer Netherlands, Dordrecht, 1996. ISBN 978-0-7923-4248-9. doi: 10. 
1007/978-94-009-1766-8. URL http://link.springer.com/10.1007/ 978-94-009-1766-8.

[61] Arnout K.H. D'Haese, Ilse De Leersnyder, Pieter Vermeir, and Arne R.D. Verliefde. On negative rejection of uncharged organic solutes in forward osmosis. J. Memb. Sci., 548:22-31, 2018. ISSN 18733123. doi: 10.1016/j.memsci.2017.11.002.

[62] V. M. M. Lobo. Mutual diffusion coefficients in aqueous electrolyte solutions (Technical Report). Pure Appl. Chem., 65(12): 2613-2640, 1993. ISSN 1365-3075. doi: 10.1351/pac199365122613. URL http://www.degruyter.com/view/j/pac.1993.65.issue-12/ pac199365122613/pac199365122613.xml.

[63] James E. McGrath, Ho Bum Park, Geoffrey M. Geise, Alyson C. Sagle, and Benny D. Freeman. Water permeability and water/salt selectivity tradeoff in polymers for desalination. J. Memb. Sci., 369(1-2):130138, 2010. ISSN 03767388. doi: 10.1016/j.memsci.2010.11.054. URL http://dx.doi.org/10.1016/j.memsci.2010.11.054. 